Saudi Journal of Medical and Pharmaceutical Sciences

Abbreviated Key Title: Saudi J Med Pharm Sci

ISSN 2413-4929 (Print) |ISSN 2413-4910 (Online)

Scholars Middle East Publishers, Dubai, United Arab Emirates

Journal homepage: http://scholarsmepub.com/sjmps/

Original Research Article

\title{
Undifferentiated Connective Tissue Disease among Patients Attending a Rheumatology Clinic
}

\author{
Dr. Akintayo Segun Oguntona, MBChB, FWACP ${ }^{1 *}$, Dr. Olabanke Arike Olatunde, MBBS, FWACP ${ }^{2}$ \\ Department of Medicine, Olabisi Onabanjo University Teaching Hospital, P.M.B. 2001 Sagamu, Ogun State, Nigeria
}

\begin{tabular}{ll}
\hline DOI: $10.36348 / S J M P S .2019 . v 05 i 12.004$ & | Received: $02.12 .2019 \mid$ Accepted: $09.12 .2019 \mid$ Published: 11.12 .2019 \\
*Corresponding author: Segun Akintayo Oguntona
\end{tabular}

*Corresponding author: Segun Akintayo Oguntona

\section{Abstract}

Background: The term undifferentiated connective tissue disease (UCTD) is used when people have symptoms and laboratory results suggestive of systemic autoimmune disease, but lack enough characteristics of a well defined connective tissue disease such as systemic lupus erythematosus, scleroderma and rheumatoid arthritis. Disease in patients with undifferentiated CTD most commonly evolve into a specific defined form while others remain unchanged. Methods: This was a prospective study of patients seen in a tertiary rheumatology clinic. The study was done over three years between July 2013 to June 2016. Patients with symptoms and signs suggestive of UCTD were included in the study. Only those with significant titre of antinuclear antibody (ANA) were included. Excluded from the study were patients with definite diagnosis of specific CTD. Socio-demographic characteristics of the patients were documented. Family history of autoimmune diseases and the time of diagnosis of UCTD were also documented. Results: Five hundred and two patients were seen in the rheumatology clinic over a period of 3 years. There were 79 patients diagnosed of connective tissue diseases with a period prevalence of $15.7 \%$. Undifferentiated connective tissue disease constituted $10.1 \%$. There were 4 females and 2 males. Patients were aged from 18 to 64 years, with a mean age of $42 \pm 5$ years. Three patients presented with lupus-like symptoms. Arthralgia was the leading symptom. ANA was uniformly positive and the patient with positive anti Ro/SSA presented with sicca symptoms. Conclusion: UCTD is not completely rare in our environment. A high index of suspicion is however needed to diagnose them.

Keywords: undifferentiated connective tissue disease (UCTD), scleroderma, symptoms, autoimmune diseases.

Copyright @ 2019: This is an open-access article distributed under the terms of the Creative Commons Attribution license which permits unrestricted use, distribution, and reproduction in any medium for non-commercial use (NonCommercial, or CC-BY-NC) provided the original author and source are credited.

\section{INTRODUCTION}

At the onset of the disease, most autoimmune connective tissue diseases are not clinically well differentiated, and this stage is called undifferentiated connective tissue disease (UCTD) [1]. About fifty percent of such patients will eventually differentiate to a define connective tissue disease (CTD). Nail-fold capillary microscopy and the autoantibody profile can help to monitor the direction of differentiation [1].

Sabo [2] in 1969 was the first to describe undifferentiated diseases. He suggested that three evolutions are possible; the disease could evolve to a definite CTD, remain undifferentiated or become intermittent undifferentiated [2]. LeRoy however in 1980 proposed the concept of UCTD to represent people who were recognized as being in the early stages of a CTD but who did not yet meet the standard criteria for a well-defined CTD [3]. Other names used earlier to describe the disease included latent lupus and incomplete lupus erythematosus.
Mosca et al., [4] in 1999 proposed preliminary classification criteria for UCTD that have become increasingly accepted and used. Those with disease duration less than three years are referred to as early UCTD while those above three years are referred to as established UCTD in the presence of positive ANA [4]. Other specific disease antibodies found are (antiRo/SSa, anti- RNP and anti-dsDNA [4].

The most common clinical features associated with UCTD are non-erosive arthritis, Raynaud's phenomenon, sclerodactyly, leukopenia. When clinical abnormalities suggestive of Sjogren's syndrome (SS) are found usually salivary gland biopsy and ocular tests are normal [5].

No mutual agreement has been reached regarding the criteria for diagnosis of UCTD [6]. UCTDs are generally treated with low-dose 
corticosteroids and anti-malarials, and very rarely with immunosuppressive drugs.

The objective of this study is to describe the clinical and serological characteristics of a cohort of patients with UCTD.

\section{METHODS AND MATERIALS}

This was a prospective study of patients seen in a tertiary rheumatology clinic. The study was done over a period of three years spanning between July 2013 to June 2016.

The inclusion criteria were consecutive patients seen at the rheumatology clinic regardless of the disease's duration that presented with signs and symptoms suggestive of UCTD and the presence of positive ANA with titre of 1:80 and above at two different occasions. Excluded from the study were patients with a well define CTD such as rheumatoid arthritis, systemic lupus erythematosus, systemic sclerosis, Sjögren's syndrome, psoriatic arthritis, antiphospholipid syndrome, systemic and inflammatory muscle disease. Patients with only one consultation and those that were negative for ANA were also excluded.

Socio-demographic characteristics of the patients were documented including age, gender, clinical presentations (arthralgia, arthritis, fever, Raynaud's phenomenon, myalgia, livedo reticularis, malar rash, hair loss, photosensitivity, weight loss, hand morning stiffness, anemia and leucopenia). Family history of autoimmune diseases and time of diagnosis of UCTD were also documented. Blood samples for serologic markers were sent for investigation.

Simple statistical analysis was used to calculate the percentage and the mean values.

\section{RESULTS}

There were 502 rheumatology patients seen over a period of 3 years (July 2013-June 2016).
Connective tissue diseases were diagnosed in 79 patients with a period prevalence of $15.7 \%$. In order of occurrence were rheumatoid arthritis $(48.1 \%)$, systemic lupus erythematosus $(15.2 \%)$, undifferentiated connective tissue disease $(10.1 \%)$, scleroderma $(7.6 \%)$, and secondary Sjogren's syndrome (7.6\%). Table-1 shows the spectrum of connective tissue diseases seen during the study. The age range of patients was 18 to 64 years, with a mean age of $42 \pm 5$ years. Most patients were in the third, fourth, and fifth decade of life. Female were predominant with $78.5 \%$ of the autoimmune population with a sex ratio of 3.6:1.

There were six patients with diagnosis of UCTD, the mean follow up of these patients after diagnosis was 3 years and five months. The most frequent clinical manifestations were arthralgia (100\%), arthritis $(83.3 \%)$, fever $(66.7 \%)$, anaemia $(66.7 \%)$, photosensitivity $(50 \%)$, leucopenia $(50 \%)$ and sicca syndrome $(33.3 \%)$. Each of the patients presented with a mean of three clinical manifestations. Three patients presented with features similar to SLE but did not fulfill the classification criteria for SLE. The lupus-like subgroup presented frequently with arthritis, cytopenias, oral ulcers and photosensitivity. The Patients with anti-SSA antibody presented with leukopenia and sicca symptoms with no objective signs of Sjogren's syndrome.

The autoimmune profile encountered were positive ANA (100\%), Anti-SS-A (16.7\%), and Anti RNP. The most frequent patterns of ANA were speckle $(50 \% ; \mathrm{n}: 3)$, homogeneous $(33.3 \% ; \mathrm{n}: 2)$ and centromere (16.7\%; n:1). No patients however had positive antidsDNA. Figure-1 shows the clinical and Laboratory features seen among the patients with UCTD.

All the patients with the diagnosis of UCTD were placed on non-steroidal anti-inflammatory drugs, corticosteroids and hydroxychloroquine. None was placed on immunosuppressive treatment.

Table-1: Connective tissue diseases spectrum seen during the study

\begin{tabular}{|l|l|l|l|}
\hline Disease & Female: n, (\%) & Male: n, (\%) & Total: n, (\%) \\
\hline Rheumatoid arthritis & $27(71.1)$ & $11(28.9)$ & $38(48.1)$ \\
\hline SLE & $11(91.7)$ & $1(8.3)$ & $12(15.2)$ \\
\hline Scleroderma & $5(83.3)$ & $1(16.7)$ & $06(7.6)$ \\
\hline Dermatomyositis & $2(100)$ & $0(0)$ & $02(2.5)$ \\
\hline Polymyositis & $2(66.7)$ & $1(33.3)$ & $03(3.8)$ \\
\hline Sjogren's syndrome & $5(83.3)$ & $1(16.7)$ & $06(7.6)$ \\
\hline $\begin{array}{l}\text { Overlap } \\
\text { * RA/Dermatomyositis }\end{array}$ & $3(100)$ & $0(0)$ & $03(3.8)$ \\
$\begin{array}{l}\text { * RA/SLE } \\
\text { *Sleroderma/Dermatomyositis }\end{array}$ & & & \\
\hline Antiphopholipid syndrome & $1(100)$ & $0(0)$ & $01(1.3)$ \\
\hline Undifferentiated & $6(75)$ & $2(25)$ & $08(10.1)$ \\
\hline Total & $\mathbf{6 2}$ & $\mathbf{1 7}$ & $\mathbf{7 9}$ \\
\hline
\end{tabular}




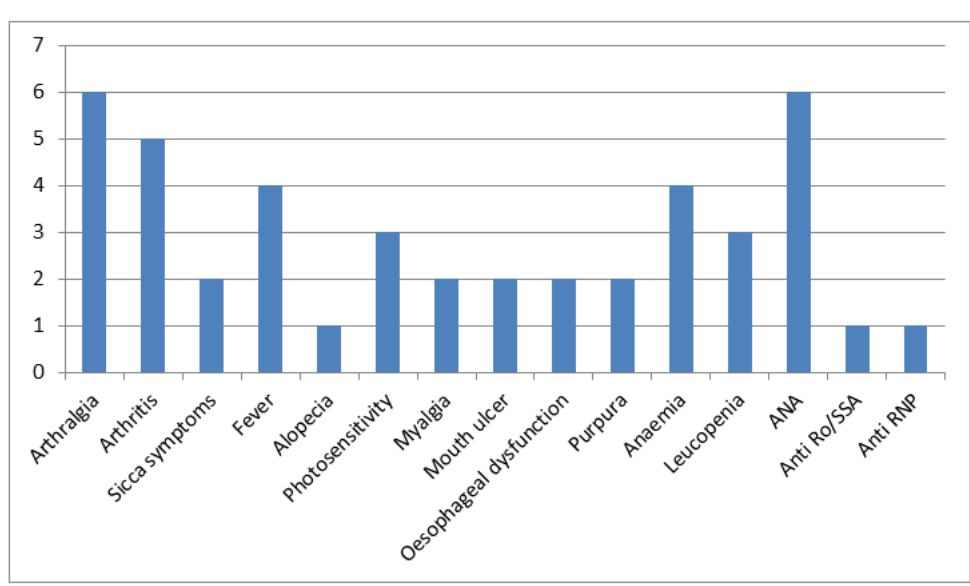

Fig-1: Clinical and Laboratory features seen among the patients with UCTD

\section{DISCUSSION}

In fully established cases, it is not difficult to make the diagnosis of diffuse connective tissue disease even with minimal investigations. In the early stages however, the diagnosis may not be obvious and these cases are often referred to as undifferentiated connective tissue disease (UCTD) [1]. UCTDs have been defined as conditions characterised by the presence of signs and symptoms suggestive of a CTD but not fulfilling the existing classification criteria. About $35 \%$ of such patients are said to have overlap syndromes while the other ones would eventually differentiate into a clinical picture that is consistent with a particular connective tissue disease ${ }^{1}$.

Disease in patients with undifferentiated CTD most commonly evolve into SLE, myositis, systemic sclerosis (SSc), primary Sjögren's syndrome (pSS), mixed connective tissue disease (MCTD), systemic vasculitis, and rheumatoid arthritis (RA) ${ }^{7}$ although there were reports that suggested most remain undifferentiated $^{7}$.

The clinical presentation of connective tissue diseases (CTDs) is extremely heterogeneous as these disorders can present with a wide variety of signs and symptoms as exemplified by our patients. Arthralgias, arthritis and fever were the commonest symptoms among our patients, while others presented with myalgias, sicca symptoms, esophageal dysfunction and alopecia. Alarcon et al., [8] in 1991 reported that about $50 \%$ of patients with CTD of duration less than 1-year had an UCTD which would subsequently either evolve to a defined CTD, resolve or remain undifferentiated.

Two patients presented with disease duration of less than three years while the remaining four patients had disease duration of over three years. Those with disease duration of less than three years belong to the group of early UCTD and those with disease duration of at least three years were said to have an established UCTD. Literature have reported that those with early UCTD patients potentially have a more clinically unstable disease, with a higher risk of evolving to a defined CTD [9]. Those with established UCTD however possibly represents a cohort of patients with a more stable clinical phenotype, which is unlikely to go into remission or evolve to a defined CTD [9].

The evolution of UCTD to defined CTDs usually occurs within the first 5 years of disease. The clinical features identified that may predict an evolution of UCTD to SLE include fever, discoid lupus, alopecia, serositis, photosensitivity [4]. Greater agreement more than that of clinical features however exists for autoantobodies such as anti-dsDNA antibodies, anti-Sm antibodies, anti-cardiolipin antibodies as well as the presence of multiple antibodies specificities [10].

Patients who do not develop a defined SLE have a mild clinical picture characterized by arthralgia, arthritis, Raynaud's phenomenon, leucopenia, anaemia, and sicca symptoms. Usually, they do not have major organ involvement (renal, neurological and the pulmonary) [5]. Nailfold capillary microscopy and the autoantibody profile can help to monitor the direction of differentiation [1].

All the patient were significantly positive for anti-nuclear antibodies (ANA). When patients present with a single antibody specificity, then the presence of positive ANA is needed to classify such patients as UCTD [11]. In such cases, anti-Ro/SSA and anti-RNP antibodies are the most commonly observed antibodies and are present in $8-30 \%$ and $10-30 \%$ of patients respectively [11]. When Anti-RNP antibodies alone is present such patients usually present with Raynaud's phenomenon with a non-specific lesions [12]. The Raynaud's phenomenon in them also appears benign without digital ulcers and pitting scars [12].

One patient had anti-Ro/SSA UCTD. She presented with arthralgia, purpura and sicca symptoms. It has been reported that $8-30 \%$ of UCTD patients have anti-Ro/SSA antibodies [13]. Cavazzana et al., [11] in 2001 reported the clinical manifestations among their 
148 UCTD patients with antibodies to Ro/SSA. The most common clinical manifestations of these patients were arthralgia (86\%), skin lesions (37\%) (livedo, purpura, urticaria, acrocyanosis, sclerodactily and teleangectasias), Raynaud's phenomenon (33\%) and sicca symptoms $(30 \%)$.

Although, our patients generally had mild disease symptoms, and according to available data, many patients with UCTD have a favorable prognosis. It is still advisable that these patients should be regularly followed up. Extrapolation of results and prognosticating UCTD has remained a problem because of variable inclusion criteria used by various groups of researchers. It therefore calls for a unified approved criteria for UCTD to be able to overcome these challenges.

\section{REFERENCES}

1. Doria, A., Mosca, M., Gambari, P. F., \& Bombardieri, S. (2005). Defining unclassifiable connective tissue diseases: incomplete, undifferentiated, or both?. The Journal of Rheumatology, 32(2), 213-215.

2. Sabo, I. (1969). The lanthanic or undifferentiated collagen disease. Hiroshima journal of medical sciences, 18(4), 259-264.

3. LeROY, E. C., \& Medsger Jr, T. A. (2001). Criteria for the classification of early systemic sclerosis. Journal of Rheumatology, 28(7), 15731576.

4. Mosca, M., \& Bombardieri, S. (2001). Undifferentiated connective tissue diseases: A new frontier in rheumatology. Current rheumatology reports, 3(4), 259-260.

5. Mosca, M., Tavoni, A., Neri, R., Bencivelli, W., \& Bombardieri, S. (1998). Undifferentiated connective tissue diseases: the clinical and serological profiles of 91 patients followed for at least 1 year. Lupus, 7(2), 95-100.

6. Vaz, C. C., Couto, M., Medeiros, D., Miranda, L., Costa, J., Nero, P., ... \& Inês, L. (2009). Undifferentiated connective tissue disease: a seven-center cross-sectional study of 184 patients. Clinical rheumatology, 28(8), 915-921.

7. Bodolay, E., Csiki, Z., Szekanecz, Z., Ben, T., Kiss, E., Szucs, G., ... \& Szegedi, G. (2003). Fiveyear follow-up of 665 Hungarian patients with undifferentiated connective tissue disease (UCTD). Clinical and experimental rheumatology, 21(3), 313-320.

8. Alarcon, G. S., Williams, G. V., Singer, J. Z., Steen, V. D., Clegg, D. O., Paulus, H. E., ... \& Willkens, R. F. (1991). Early undifferentiated connective tissue disease. I. Early clinical manifestation in a large cohort of patients with undifferentiated connective tissue diseases compared with cohorts of well established connective tissue disease. The Journal of rheumatology, 18(9), 1332-1339.

9. Danieli, M. G., Fraticelli, P., Franceschini, F., Cattaneo, R., Farsi, A., Passaleva, A., ... \& Sabbadini, M. G. (1999). Five-year follow-up of 165 Italian patients with undifferentiated connective tissue diseases. Clinical and experimental rheumatology, 17, 585-592.

10. Vila, L. M., Mayor, A. M., Valent, A., Garc, M., \& Vila, S. (2000). Clinical outcome and predictors of disease evolution in patients with incomplete lupus erythematosus. Lupus, 9(2), 110-115.

11. Cavazzana, I., Franceschini, F., Belfiore, N., Quinzanini, M., Caporali, R., Calzavara-Pinton, P., ... \& Montecucco, C. (2001). Undifferentiated connective tissue disease with antibodies to Ro/SSa: clinical features and follow-up of 148 patients. Clinical and experimental rheumatology, 19(4), 403-410.

12. Doria, A., Mosca, M., Gambari, P. F., \& Bombardieri, S. (2005). Defining unclassifiable connective tissue diseases: incomplete, undifferentiated, or both?. The Journal of Rheumatology, 32(2), 213-215.

13. Mosca, M., Neri, R., Bencivelli, W., Tavoni, A., \& Bombardieri, S. (2002). Undifferentiated connective tissue disease: analysis of 83 patients with a minimum followup of 5 years. The Journal of Rheumatology, 29(11), 2345-2349. 